\title{
Photographic Archives of Ukrainian Observatories: Digitizing a Heritage
}

\author{
Ludmila Pakuliak $^{1}$, Lilia Kazantseva ${ }^{2}$, Natalia Virun ${ }^{3}$ \\ and Vitaly Andruk ${ }^{1}$ \\ ${ }^{1}$ Main Astronomical Observatory, NAS of Ukraine, Kyiv, 03680 Ukraine \\ email: pakuliak@mao.kiev.ua \\ ${ }^{2}$ Astronomical Observatory of Kyiv National University, Kyiv, 04053 Ukraine \\ email: likaz@observ.univ.kiev.ua \\ ${ }^{3}$ Astronomical Observatory of Lviv National University, Lviv, 79005 Ukraine \\ email: virun@astro.franko.lviv.ua
}

\begin{abstract}
We describe a key project of the Ukrainian Virtual Observatory (UkrVO), namely, a collaboration to digitize the large collections of photographic plates that had been exposed during more than 100 years at Ukrainian observatories, and to combine the digitized images with CCD archives to form the UkrVO Joint Digital Archive. The application of flatbed scanners for digitizing plates is discussed.
\end{abstract}

Keywords. astronomical data bases: miscellaneous

\section{Plate Collections of Ukrainian Observatories}

The archives of photographic observations at three observatories of Ukraine - at Kyiv, Lviv and Odessa Universities - are significant through both their size and their age; the collections are very extensive, and cover the period from the late- $19^{\text {th }}$ century to the mid- $20^{\text {th }}$ century. A small part of the plates from that period may have been dispersed when observing activities were severely disrupted during WWI and WWII and several eastern European observatories lost major parts of their plate collections. The loss of a collection also means the loss of information about the observing methods used.

The history of progress in observational techniques has a number of dimensions, cultural as well as scientific. Observing involved Hartmann or hexagonal diaphragms, tubular, wedge or Fesenkov photometers, hypersensitizing in special chemical solutions, and many other methods and processes that have now been relegated to the status of littleknown techniques of historic interest only. Unfortunately, it is difficult to recover complete information about the images that were observed, as logs and other records were often lost. Nevertheless, each series of historic photographic observations is worthy of careful attention and study. We therefore included the preservation of historical observational archives within the framework of the Ukrainian Virtual Observatory project without segregation into scientific and historical sections.

\subsection{The AO LNU collection}

The Astronomical Observatory of Lviv National University (AO LNU) made photographic observations from 1939 to 1976; just a few were also made between 1936 and 1939. The instruments that were used included a camera with a Zeiss triplet lens $(\mathrm{D} / \mathrm{F}=$ $100 / 500 \mathrm{~mm})$, a Mertz refractor, an astro-camera $(\mathrm{D} / \mathrm{F}=140 / 700 \mathrm{~mm})$ and a Zeiss refractor $(\mathrm{D} / \mathrm{F}=130 / 2400 \mathrm{~mm})$. Up to the 1950 s the plates were measured for photovisual 
photometry with a Schilt photometer. Part of the collection had never been reduced at all, or processed only partially.

\subsection{The $A O K N U$ collection}

The photographic collection of the Astronomical Observatory of Kyiv National University (AO KNU) has over 20,000 plates; it covers the period 1898-1996, and is sub-divided into more than 200 series. $65 \%$ of the collection is on glass; the rest is on large-format film. Most of the series include images of photometric standard fields obtained on the same dates as target images, or other exposures allowing different methods of calibration (out-of-focus images for use in conjunction with a photo-tube, a photometric wedge, the Sabattier effect, or the method of equi-densities for photometry of extended objects). In 2010 the first attempts were made to provide open access to the historical (pre1950) section of the archive. Test digitization of about 100 plates gave very encouraging precisions: $0^{\prime \prime} .1$ in position and 0.07 in magnitude.

\section{The Joint Digital Archive of the UkrVO}

The Joint Digital Archive (JDA) of photographic and CCD observations was conceived as a key project of the UkrVO (Vavilova et al. 2010). Digitizing Ukraine's photographic plates started in 2008. The JDA is being required to include digitized photographic images from at least six Ukrainian observatories. The plates had been exposed in more than 20 different instruments, so it means that every "publisher" of digitized images has to transform the data to a common standard before placing them in a shared database.

Flatbed scanners have recently become popular as digitizers for photographic plate archives. The quality of the output from digitization by commercial scanners has caused, and still causes, considerable concern. Nevertheless, commercial scanners are the most widely available appliances for relatively rapid digitization work, and applying the proper scan procedures and algorithms to the digitized images yields the best accuracy that can be achieved for a given appliance and given observational material.

Processed files from the digital archives of MAO NASU, AO LNU and AO KNU are mounted on the computer cluster of the Main Astronomical Observatory NAS, which are shared resources. A database of the Golosiiv plate archive has been available there via open access (DBGPA, http://www.gua.db.ukr-vo.org) since 2003, and serves as a test area for JDA development and software upgrades for data access. To date that JDA prototype holds digitized data from four plate collections, including their historical sections.

Digitizing the plates is carried out with two models of flatbed scanner (Table 1). Two types of digitized images are derived. For the highest-quality plates, high-resolution scans are made twice, one with the plate rotated clockwise through $90^{\circ}$. The TIFF output images have a dynamic range of 16 bits in grey scale and a resolution of $1200 \mathrm{dpi}$. The maximum linear dimension is 13,000 pixels. A different type of scanned image is made for "previews" - rapid preliminary visualization of the content and quality of the material. Most of the plates scanned in that way are wide-field plates, like the ones included in the WFPDB (Tsvetkova et al. 2008). In the preview images any ink marks or writing are preserved as they may have historic value (especially when the plates are nearly a century old), and are only subsequently cleaned off. JPEG previews are scanned with a grey-scale of 8 bits, or 24 bits for a coloured image, and a resolution of 300-1200 dpi. The maximum linear dimension is 1,200 pixels along the larger side for plates of any size. 


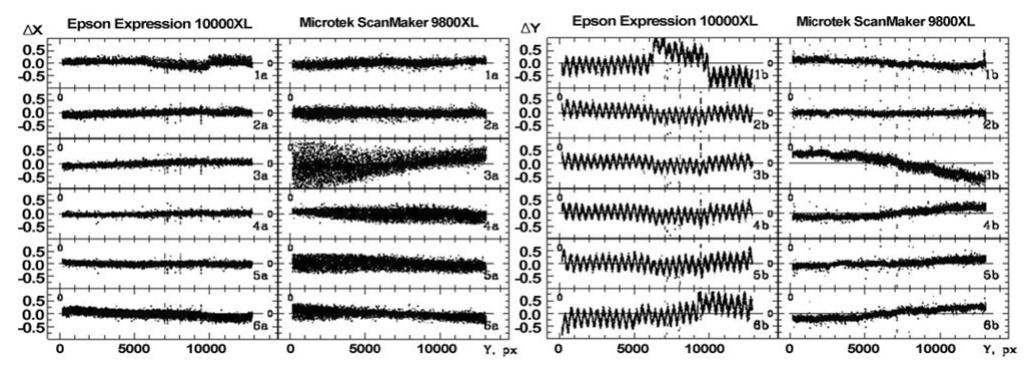

Figure 1. Trends of individual deviations of coordinates $\Delta \mathrm{X}, \Delta \mathrm{Y}$ from the average values

Table 1. Comparing accuracies of performance for different image resolutions.

\begin{tabular}{|c|c|c|c|c|c|c|c|c|c|c|}
\hline & Epso & n Ex & pressi & ion 100 & $00 X \mathrm{~L}$ & | Micr & rotek & Scan & Maker & $9800 \mathrm{XL}$ \\
\hline dpi & 300 & 600 & | 900 | & 1200 & 1600 & $|300|$ & 600 & $|900|$ & 1200 & 1600 \\
\hline $\begin{array}{l}\sigma_{m}\left({ }^{m}\right) \\
\sigma_{\alpha}\left({ }^{\prime \prime}\right) \\
\sigma_{\delta}\left({ }^{\prime \prime}\right)\end{array}$ & $\begin{array}{l}0.18 \\
1.12 \\
1.17\end{array}$ & $\begin{array}{l}0.22 \\
0.69 \\
0.73\end{array}$ & $\left|\begin{array}{l}0.24 \\
0.62 \\
0.63\end{array}\right|$ & $\begin{array}{l}0.22 \\
0.49 \\
0.54\end{array}$ & $\begin{array}{l}0.22 \\
0.41 \\
0.46\end{array}$ & $\left|\begin{array}{l}0.22 \\
1.84 \\
2.04\end{array}\right|$ & $\begin{array}{l}0.34 \\
1.27 \\
1.52\end{array}$ & $\begin{array}{l}0.40 \\
0.90 \\
1.18\end{array}$ & $\begin{array}{l}0.35 \\
0.78 \\
1.07\end{array}$ & $\begin{array}{l}0.36 \\
0.63 \\
0.98\end{array}$ \\
\hline
\end{tabular}

\section{Comparing Scanners to Estimate their Accuracy}

In order to estimate the photometric and positional accuracy of the scanners, a set of six sequential scans was made of the same plate at $1200 \mathrm{dpi}(21.17 \mu \mathrm{m} / \mathrm{pixel})$. Averages of the six scans were derived, for each scanner and every object. The total number of recorded objects was $\sim 100,000$, though selecting objects with $6<V<13$ from the TYCHO-2 Catalogue reduced that number to 6000 . Special attention was paid to (a) systematic trends (and their elimination) produced by the scanner, (b) correct separation of images on multi-exposure plates, and (c) the effects of different resolution (for which another set of images with resolutions of 300-1600 dpi was obtained).

The results showed that the optimum resolution for both instruments was 1200 dpi. Trends of individual positional deviations $(\Delta \mathrm{X}, \Delta \mathrm{Y})$ from the average values are illustrated in Fig. 1. After removing systematic trends, the rms errors were $\sigma_{X, Y}= \pm 0.03-$ 0.06 pixels, meaning that $\sigma_{\alpha, \delta} \leqslant \pm 0^{\prime \prime} .1$ for both scanners. The values of $\left(\sigma_{m}\right)$, the rms photometric errors, were $\leqslant \pm 0.015 \mathrm{mag}$, and $\sigma_{B} \leqslant \pm 0.03 \mathrm{mag}$. for both digitizers too. Details of the accuracy and precision for different resolutions are given in Table 1 . In the light of those results, an Epson Expression model was selected for making high-resolution images, and a Microtek one for preview digitization.

At the time of writing, the number of digitized images in the MAO NASU archive is approaching 4000, and there are nearly 2500 in the AO LNU archive. Digitization of the AO KNU archive has just commenced.

The rationale and methods adopted for digitizing these historical components of an archive are described on our Website at http://ukr-vo.org/history/index.php?b1\&4 and http://ukr-vo.org/history/index.php?b2\&4. Placing these descriptions in the public domain provides a resource that can be used for educational and museum activities, in particular in relation to the history of the science of astronomy in the Ukraine.

\section{References}

Vavilova, I. B., Pakuliak, L. K., \& Protsyuk, Yu.I. 2010, Kosm. Nauka Tekhn., 16, 62

Tsvetkova, K. P., Tsvetkov, M. K., Sergeeva, T. P., \& Sergeev, A. V. 2009, Kin. E Phys.of Celest. Bodies, 25, 402 